\title{
Exploration and Customization of FPGA-Based Soft Processors
}

\author{
Peter Yiannacouras, Student Member, IEEE, J. Gregory Steffan, Member, IEEE, and \\ Jonathan Rose, Senior Member, IEEE
}

\begin{abstract}
As embedded systems designers increasingly use field-programmable gate arrays (FPGAs) while pursuing single-chip designs, they are motivated to have their designs also include soft processors, processors built using FPGA programmable logic. In this paper, we provide: 1) an exploration of the microarchitectural tradeoffs for soft processors and 2) a set of customization techniques that capitalizes on these tradeoffs to improve the efficiency of soft processors for specific applications. Using our infrastructure for automatically generating soft-processor implementations (which span a large area/speed design space while remaining competitive with Altera's Nios II variations), we quantify tradeoffs within soft-processor microarchitecture and explore the impact of tuning the microarchitecture to the application. In addition, we apply a technique of subsetting the instruction set to use only the portion utilized by the application. Through these two techniques, we can improve the performance-per-area of a soft processor for a specific application by an average of $25 \%$.
\end{abstract}

Index Terms-Customization, design space exploration, field-programmable gate array (FPGA)-based soft-core processors, processor generator.

\section{INTRODUCTION}

$\mathbf{F}$ IELD-PROGRAMMABLE gate array (FPGA) vendors now support processors on their FPGA devices to allow complete systems to be implemented on a single programmable chip. Although some vendors have incorporated fixed hard processors on their FPGA die, there has been a significant adoption of soft processors [1], [2] which are constructed using the FPGA's programmable logic itself. When a soft processor can meet the constraints of a portion of a design, the designer has the advantage of describing that portion of the application using a high-level programming language such as $\mathrm{C} / \mathrm{C}++$. More than $16 \%$ of all FPGA designs [3] contain soft processors, even though a soft processor cannot match the performance, area, and power of a hard processor [4]. FPGA platforms differ vastly from transistor-level platforms-hence, previous research in microprocessor architecture is not necessarily applicable to soft processors implemented on FPGA fabrics, and we are therefore motivated to revisit processor architecture in an FPGA context.

Soft processors are compelling because of the flexibility of the underlying reconfigurable hardware in which they are implemented. This flexibility leads to two important areas of

Manuscript received March 15, 2006; revised July 6, 2006. This work was supported in part by NSERC and in part by the Altera Corporation. This paper was recommended by Associate Editor A. DeHon.

The authors are with the Edward S. Rogers Sr. Department of Electrical and Computer Engineering, University of Toronto, Toronto, ON M5S 364 Canada (e-mail: yiannac@eecg.utoronto.ca; steffan@eecg.utoronto.ca; jayar@eecg. utoronto.ca).

Digital Object Identifier 10.1109/TCAD.2006.887921 investigation for soft-processor architecture that we address in this paper. First, we want to understand the architectural tradeoffs that exist in FPGA-based processors, by exploring a broad range of high-level microarchitectural features such as pipeline depth and functional unit implementation. Our longterm goal is to move toward a computer aided design (CAD) system, which can decide the best soft-processor architecture for given area, power, or speed constraints. Second, we capitalize on the flexibility of the underlying FPGA by customizing the soft-processor architecture to match the specific needs of a given application: 1) We improve the efficiency of a soft processor by eliminating the support for instructions that are unused by an application. 2) We also demonstrate how microarchitectural tradeoffs can vary across applications, and how these also can be exploited to improve efficiency with applicationspecific soft-processor designs. Note that these optimizations are orthogonal to implementing custom instructions that target custom functional units/coprocessors, which is beyond the scope of this paper.

To facilitate the exploration and customization of the softprocessor architecture, we have developed the soft-processor rapid exploration environment (SPREE) to serve as the core of our software infrastructure. SPREE's ability to generate synthesizable register-transfer level (RTL) implementations from higher level architecture descriptions allows us to rapidly explore the interactions between architecture and both hardware platform and application, as presented in two previous publications [5], [6]. This paper unifies our previous results and includes the exploration of a more broad architectural space.

\section{A. Related Work}

Commercial customizable processors are available from Tensilica [7] for application-specific integrated circuits (ASICs), Stretch [8] as an off-the-shelf part, and others which allow designers to tune the processor with additional hardware instructions to better match their application requirements. Altera Nios [1] and Xilinx Microblaze [2] are processors meant for FPGA designs which also allow customized instructions or hardware, and are typically available in only a few microarchitectural variants.

Research in adding custom hardware to accelerate a processor has shown a large potential. The GARP project [9] can provide $2-24 \times$ speedup for some microkernels using a custom coprocessor. More recent work [10] in generating custom functional units while considering communication latencies between the processor and custom hardware can achieve $41 \%$ 
speedup and similar energy savings. Dynamic custom hardware generation [11] delivers $5.8 \times$ speedups and $57 \%$ less energy. However, these approaches, which transform critical code segments into custom hardware, can also benefit from the customizations in our own work: 1) Additional real estate can be afforded for custom hardware by careful architecting of the processor and appropriate subsetting of the required instruction set using the SPREE. 2) Once the custom hardware is implemented, the criticality is more evenly distributed over the application complicating the identification of worthwhile custom hardware blocks. As a result, rearchitecting is required for the new instruction stream if more efficiency is sought, a task SPREE is designed to facilitate.

The CUSTARD [12] customizable threaded soft processor is an FPGA implementation of a parameterizable core supporting the following options: different number of hardware threads and types, custom instructions, branch delay slot, load delay slot, forwarding, and register file size. While the available architectural axes seem interesting, the results show large overheads in the processor design. Clock speed varied only between 20 and $30 \mathrm{MHz}$ on the $0.15-\mu \mathrm{m} \mathrm{XC2V2000,} \mathrm{and} \mathrm{the} \mathrm{single-threaded}$ base processor consumed 1800 slices while the commercial Microblaze typically consumes less than 1000 slices on the same device.

PEAS-III [13], EXPRESSION [14], LISA [15], MADL [16], RDLC [17], and many others use architecture description languages (ADLs) to allow designers to build a specific processor or explore a design space. However, we notice three main drawbacks in these approaches for our purposes: 1) The verbose processor descriptions severely limit the speed of design space exploration. 2) The generated RTL, if any, is often of poor quality and does not employ necessary special constructs which encourage efficient FPGA synthesis. 3) Most of these systems are not readily available or have had only subcomponents released. To the best of our knowledge, no real exploration was done beyond the component level [13], [14] and certainly not done in a soft-processor context.

Gray has studied the optimization of CPU cores for FPGAs [18]. In those processors synthesis and technology mapping tricks are applied to all aspects of the design of a processor from the instruction set to the architecture. While that work documents constructs used for efficient synthesis, our work is somewhat orthogonal: We are not focused on customizing the processor to its hardware platform and discovering "free" FPGA optimizations, but rather, we assume (and have created) a variety of "good" FPGA-optimized hardware configurations and explore their different benefits to different applications. Once the best configuration is known, one can apply synthesis tricks at the component level on top of our application-level customizations to further improve the efficiency of SPREE generated processors.

\section{Generating Soft Processors With SPREE}

The evaluations presented in this paper use the SPREE [5], a system we developed to allow the fast and easy generation of a large number of soft-processor designs. In particular, the SPREE takes as input a high-level text-based description of

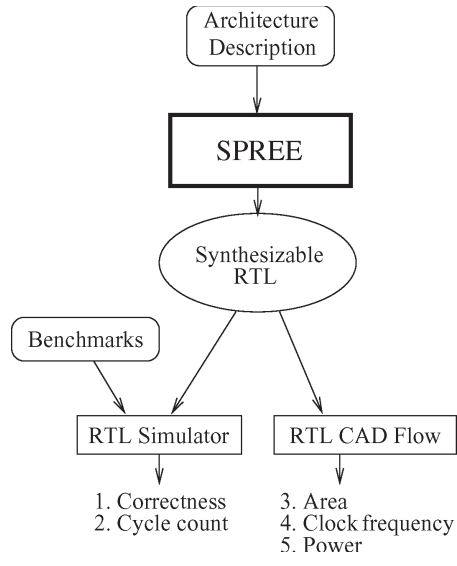

Fig. 1. Overview of the SPREE system.

the target instruction-set architecture (ISA) and datapath and generates an RTL description of a working soft processor.

Fig. 1 shows an overview of the SPREE system. Taking a high-level description of an architecture as input, the SPREE automatically generates synthesizable RTL (in Verilog). We then simulate the RTL description on benchmark applications to both ensure correctness of the processor and to measure the total number of cycles required to execute each application. The RTL is also processed by CAD tools, which accurately measure the area, clock frequency, and power of the generated soft processor. The following discussion describes how the SPREE generates a soft processor in more detail-complete descriptions of SPREE are available in previous publications [5], [19], [20].

\section{A. Input: Architecture Description}

The input to SPREE is a description of the desired processor, composed of textual descriptions of both the target ISA and the processor datapath. Each instruction in the ISA is described as a directed graph of generic operations (GENOPs), such as ADD, XOR, PCWRITE, LOADBYTE, and REGREAD. The graph indicates the flow of data from one GENOP to another required by that instruction. The SPREE provides a library of basic components (e.g., a register file, adder, sign extender, instruction fetch unit, forwarding line, and more). A processor datapath is described by the user as an interconnection of these basic components. As we describe in the following, SPREE ensures that the described datapath is capable of implementing the target ISA.

The decision to use a structural architectural description in SPREE reflects our goal of efficient implementation. Structural descriptions provide users with the ability to better manage the placement of all components including registers and multiplexers in the datapath. This management is crucial for balancing the logic delay between registers to achieve fast clock speeds. By analyzing the critical path reported by the CAD tool, users can identify the components which limit the clock frequency and take one of three actions: 1) reducing the internal logic delay of a component, for example, making a unit complete in two cycles instead of one; 2) moving some of the logic (such as multiplexers and sign extenders) from the high delay path into neighboring pipeline stages to reduce the amount of logic in 
the high delay path; and 3) adding nonpipelined registers in the high delay path causing a pipeline stall. The latter two of these actions depends critically on this ability to manually arrange the pipeline stages, referred to as retiming, which is difficult for modern synthesis tools because of the complexity in the logic for controlling the pipeline registers. Without a good ability to optimize delay, we risk making incorrect conclusions based on poor implementations. For example, one might conclude that the addition of a component does not impact clock frequency because the impact is hidden by the overhead in a poorly designed pipeline. For this reason, an architectural exploration in academia has traditionally neglected the clock-frequency considerations.

\section{B. Generating a Soft Processor}

From the above inputs, SPREE generates a complete Verilog RTL model of the desired processor in three phases: 1) datapath verification; 2) datapath instantiation; and 3) control generation. In the datapath verification phase, the SPREE compares the submitted ISA description and datapath description, ensuring that the datapath is functionally capable of executing all of the instructions in the ISA description. The datapath instantiation phase automatically generates multiplexers for sinks with multiple sources and eliminates any components that are not required by the ISA. Finally, the control-generation phase implements the control logic necessary to correctly operate the datapath, and it emits the Verilog descriptions of the complete processor design. Control generation is where the SPREE environment adds the most value since it automatically handles multicycle and variable cycle functional units, the select signals for multiplexers, the operation codes for each functional unit, the interlocking between pipeline stages, and the complexities in branching including the proper handling of branch delay slots.

\section{Limitations}

There are several limitations to the scope of soft-processor microarchitectures that we study in this paper. For now, we consider simple in-order issue processors that use only onchip memory and, hence, have no cache-since the relative speeds of memory and logic on a typical FPGA are much closer than for a hard processor chip, we are less motivated to explore an on-chip memory hierarchy for soft processors. The largest FPGA devices have more than one megabyte of on chip memory which is adequate for the applications that we study in this paper-however, in the future, we do plan to broaden our application base to those requiring off-chip RAM, which would motivate caches. We do not yet include support for dynamic branch prediction, exceptions, or operating systems. Finally, in this paper, we do not add new instructions to the ISA, we restrict ourselves to a subset of MIPS-I and have not modified the compiler, with the exception of evaluating software-versushardware support for multiplication due to the large impact of this aspect on cycle time and area.

The millions of instructions per second (MIPS) instruction set was chosen tentatively due to the abundance of available software tools (compilers, instruction simulators, etc.) available for it and because of its striking similarities with the Nios II ISA. The appropriateness of a $\mathrm{C}$ sequential programming model and sequential Harvard architecture model for soft processors is not evaluated in this paper. In addition, we have not evaluated other ISAs, but we expect similar reduced instruction-set computer (RISC) architectures to follow the same trends.

\section{EXPERIMENTAL FRAMEWORK}

Having described the SPREE system, we now describe our framework for measuring and comparing the soft processors that it produces. We present methods for verifying the processors, employing FPGA CAD tools, and measuring and comparing soft processors. Also, we discuss the benchmark applications that we use to do so.

\section{A. Processor Verification}

SPREE verifies that the datapath is capable of executing the target ISA-however, we must also verify that the generated control logic and the complete system function correctly. We implement trace-based verification by using a cycle-accurate industrial RTL simulator (Modelsim) that generates a trace of all writes to the register file and memory as it executes an application. We compare this trace to one generated by MINT [21] (a MIPS instruction set simulator) and ensure that the traces match. The SPREE automatically generates test benches for creating traces and also creates debug signals to ease the debugging of pipelined processors.

\section{B. FPGAs, CAD, and Soft Processors}

While the SPREE itself emits Verilog which is synthesizable to any target FPGA architecture, we have selected Altera's Stratix [22] device for performing our FPGA-based exploration. The library of processor components thus targets Stratix I FPGAs. We use Quartus II v4.2 CAD software for synthesis, technology mapping, placement, and routing. We synthesize all designs to a Stratix EP1S40F780C5 device (a middle-sized device in the family, with the fastest speed grade) and extract and compare area, clock frequency, and power measurements as reported by Quartus.

We have taken the following measures to counteract variation caused by the nondeterminism of CAD tool output: 1) We have coded our designs structurally to avoid the creation of inefficient logic from behavioral synthesis; 2) we have experimented with optimization settings and ensured that our conclusions do not depend on them; and 3) for the area and clock frequency of each soft-processor design, we determine the arithmetic mean across ten seeds (different initial placements before placement and routing) so that we are $95 \%$ confident that our final reported value is within $2 \%$ of the true mean.

The difference between ASIC and FPGA platforms is large enough that we are motivated to revisit the microarchitectural design space in an FPGA context. However, FPGA devices differ among themselves. Across device families and vendors, the resources and routing architecture on each FPGA vary greatly. We have focused on a single FPGA device, the Altera Stratix, to enable efficient synthesis through device-specific 
optimizations. Our hypothesis is that in spite of differences in FPGA architecture, the conclusions drawn about soft-processor architecture will be transferable between many FPGA families. In the future, we plan to investigate this across a range of different FPGA families. For now, we have migrated from Stratix I to Stratix II and observed that there is some noise in the results, but most of the conclusions still hold.

\section{Metrics for Measuring Soft Processors}

To measure area, performance, and efficiency, we must decide on an appropriate set of specific metrics. For an FPGA, one typically measures area by counting the number of resources used. In Stratix, the main resource is the logic element (LE), where each LE is composed of a four-input lookup table (LUT) and a flip-flop. Other resources, such as the hardware multiplier block and memory blocks, can be converted into an equivalent number of LEs based on the relative areas of each in silicon. ${ }^{1}$ Hence, we report the actual silicon area of the design including routing in terms of equivalent LEs.

To measure the performance, we account for both the clock frequency and instructions-per-cycle (IPC) behavior of the architecture by measuring either wall clock time or instruction throughput per second in MIPS. Reporting either clock frequency or IPC alone can be misleading; and in this paper, we have the unique ability to capture both accurately. To be precise, we multiply the clock period (determined by the Quartus timing analyzer after routing) with the number of cycles spent executing the benchmark to attain the wall-clock-time execution for each benchmark. Dividing the total number of instructions by the wall clock time gives instruction throughput.

We measure the efficiency by computing the performance gained in the instruction throughput per unit area in LEs; thus, it is measured in units of MIPS/LE. This metric is analogous to the inverse of area-delay product, an often used but debatable metric for simultaneously capturing area and speed. The best processor for a specific application depends on that application's weighting of area and speed (as an extreme, some application's might care only about one and not the other), this metric assumes a balanced emphasis on both area and speed.

\section{Benchmark Applications}

We measure the performance of our soft processors using 20 embedded benchmark applications from four sources (as summarized in Table I). Some applications operate solely on integers, and others on floating point values (although for now, we use only software floating point emulation); some are compute intensive, while others are control intensive. Table I also indicates any changes we have made to the application to support measurement, including reducing the size of the input data set to fit in on-chip memory (d) and decreasing the number of iterations executed in the main loop to reduce simulation times (i). Additionally, all file and other I/O were removed since we do not yet support an operating system.

\footnotetext{
${ }^{1}$ The relative area of these blocks was provided by Altera [23] and is proprietary.
}

TABLE I

BENCHMARK APPLICATIONS EVALUATED

\begin{tabular}{|c|c|c|c|}
\hline Source & Benchmark & Modified & $\begin{array}{r}\text { Dyn. Instr. } \\
\text { Counts }\end{array}$ \\
\hline \multirow[t]{8}{*}{ MiBench [24] } & BITCNTS & di & 26,175 \\
\hline & CRC32 & $\mathrm{d}$ & 109,414 \\
\hline & QSORT $^{*}$ & $\mathrm{~d}$ & 42,754 \\
\hline & SHA & d & 34,394 \\
\hline & STRINGSEARCH & d & 88,937 \\
\hline & FFT $^{*}$ & di & 242,339 \\
\hline & DIJKSTRA* & d & 214,408 \\
\hline & PATRICIA & di & 84,028 \\
\hline \multirow[t]{9}{*}{ XiRisc [25] } & BUBBLE_SORT & & 1,824 \\
\hline & $\mathrm{CRC}$ & & 14,353 \\
\hline & DES & & 1,516 \\
\hline & FFT $^{*}$ & & 1,901 \\
\hline & FIR ${ }^{*}$ & & 822 \\
\hline & QUANT* ${ }^{*}$ & & 2,342 \\
\hline & IQUANT* & & 1,896 \\
\hline & TURBO & & 195,914 \\
\hline & VLC & & 17,860 \\
\hline Freescale [26] & DHRY* & i & 477,564 \\
\hline \multirow[t]{2}{*}{ RATES [27] } & GOL & di & 129,750 \\
\hline & $\mathrm{DCT}^{*}$ & di & 269,953 \\
\hline
\end{tabular}

\section{COMPARISON With NiOS II VARIATIONS}

To ensure that our generated designs are indeed interesting and do not suffer from prohibitive overheads, we have selected Altera's Nios II version 1.0 family of processors for comparison. Nios II has three mostly unparameterized variations: Nios II/e, a small unpipelined 6 cycles per instruction (CPI) processor with serial shifter and software multiplication; Nios II/s, a five-stage pipeline with multiplier-based barrel shifter, hardware multiplication, and instruction cache; and Nios II/f, a large six-stage pipeline with dynamic branch prediction, and instruction and data caches.

We have taken several measures to ensure that the comparison against the Nios II variations is as fair as possible. We have generated each of the Nios processors with memory systems identical to those of our designs: Two 64-KB blocks of RAM are used for separate instruction and data memories. We do not include cache area in our measurements, although some logic required to support the caches will inevitably count toward the Nios II areas. The Nios II instruction set is very similar to the MIPS-I ISA with some minor modifications in favor of Nios (for example, the Nios ISA has no tricky branch delay slots)-hence Nios II and our generated processors are very similar in terms of ISA. Nios II supports exceptions and operating system instructions, which are so far ignored by SPREE, meaning SPREE processors save on the hardware costs in implementing these. Finally, like Nios II, we also use gcc as our compiler, although we did not modify any machine specific parameters nor alter the instruction scheduling. Despite these differences, we believe that comparisons between Nios II and our generated processors are relatively fair, and that, we can be confident that our architectural conclusions are sound.

For this experiment, we generated all three Nios II variations in the manner outlined previously, and we also generated 


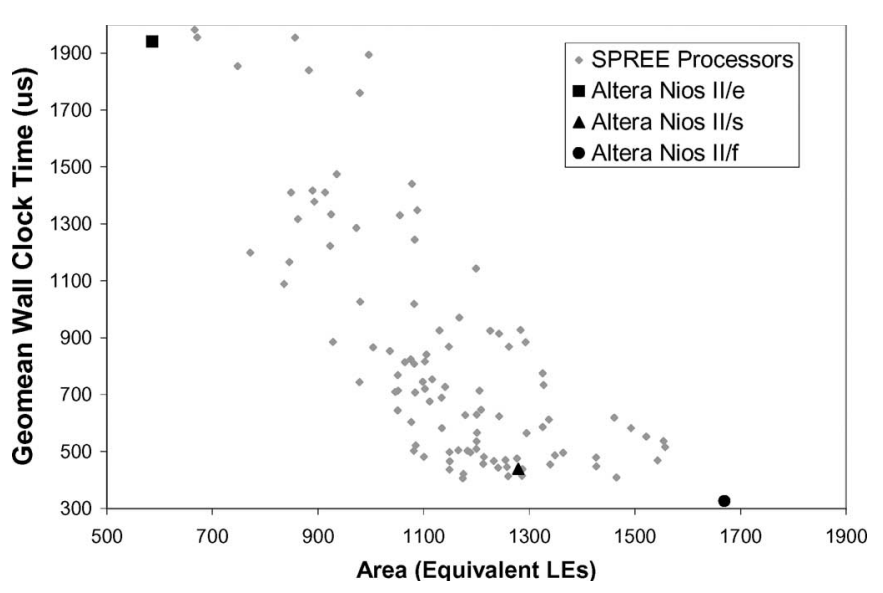

Fig. 2. Comparison of our generated designs versus the three Altera Nios II variations.

several different SPREE processors that varied in their pipelines and functional units. All of these processors are benchmarked using the same applications from our benchmark set and synthesized to the same device. Their area and performance are measured using the measurement methodology outlined in the previous section.

Fig. 2 illustrates our comparison of SPREE generated processors to the commercial Altera Nios II variations in the performance-area space. SPREE's generated processors span the design space between Nios II variations, while allowing more fine-grained microarchitectural customization. The figure also shows that SPREE processors remain competitive with the commercial Nios II. In fact, one of our generated processors is both smaller and faster than the Nios II/s-hence, we examine that processor in greater detail.

The processor of interest is an 80-MHz three-stage pipelined processor, which is $9 \%$ smaller and $11 \%$ faster in wall clock time than the Nios II/s, suggesting that the extra area used to deepen Nios II/s's pipeline succeeded in increasing the frequency, but increased overall wall clock time. The generated processor has full interstage forwarding support and hence no data hazards, and suffers no branching penalty because of the branch delay slot instruction in MIPS. The CPI of this processor is 1.36 , whereas the CPIs of Nios II/s and Nios II/f are 2.36 and 1.97, respectively. However, this large gap in CPI is countered by a large gap in clock frequency: Nios II/s and Nios II/f achieve clock speeds of 120 and $135 \mathrm{MHz}$, respectively, while the generated processor has a clock of only $80 \mathrm{MHz}$. These results demonstrate the importance of evaluating the wall clock time over the clock frequency or CPI alone, and that faster frequency is not always better.

\section{ExPloring Soft-Processor Architecture}

In this section, we employ the SPREE soft-processor generation system to explore the architectural terrain of soft processors when implemented and executed on FPGA hardware. The goal here is to seek and understand tradeoffs that may be employed to tune a processor to its application. We vary a number of core architectural parameters and measure their effects on the processor. Additionally, we attempt to attribute nonintuitive exploratory results to fundamental differences of an FPGA versus an ASIC: 1) Multiplexing is costly - their high number of inputs and low computational density means they generally map poorly to LUTs. 2) Multiplication is efficient-FPGA vendors now include dedicated multiplier circuitry meaning performing multiplication can be done comparatively more efficient than in an ASIC (relative to other logic on the same fabric). 3) Storage is cheap-with every LUT containing a flip-flop and dedicated memory blocks scattered throughout the device, storage space is abundant in modern FPGAs. 4) Memories are fast-the dedicated memories on the device can be clocked as fast as a simple binary counter [28]. 5) More coarse-grained progression of logic levels-in an FPGA, a LUT is considered a single level of logic but, in fact, can encompass several levels of logic worth of ASIC gates; however, a steep intercluster routing penalty is paid for connecting multiple LUTs.

\section{A. Functional Units}

The largest integer functional units in a soft processor are the shifter, the multiplier, and the divider. The divider is excluded from any study as it is too large (measured up to 1500 LEs compared to 1000 LEs for the rest of the processor), and it seldomly appears in the instruction streams of our benchmarks (only four benchmarks contain divides, but in each case, they make up less than half a percent of the instruction stream). Thus, we eliminate the divider unit and support division using a software subroutine. We hence focus on only the shifter and the multiplier.

1) Shifter Implementation: The shifter unit can be implemented in one of four ways: in a shift register which requires one clock cycle for every bit shifted, in LUTs as a tree of multiplexers, in the dedicated multipliers as a separate functional unit, or in the dedicated multipliers as a shared multiplier/shifter unit as used by Metzgen [29]. We implement each of these in four different pipelines and contrast the different processors with respect to their area, performance, and energy on our set of benchmarks.

With respect to area, the processors with shared multiplierbased shifter are 186 equivalent LEs smaller than the LUT based, and 147 equivalent LEs smaller than the unshared multiplier-based shifter. The performances of the three were very similar (save for minor variations in clock frequency). This leads us to conclude that because of the large area savings and matched performance, this implementation is generally favorable over both the LUT-based shifter and the unshared multiplier-based shifter.

Fig. 3 shows the performance of all benchmarks on a threestage pipeline with either the serial shifter or multiplier-based shifter. The processor with serial shifting is smaller by $64 \mathrm{LEs}$, but it also pays a heavy performance penalty when the shifter is used frequently. For example, the CRC, TURBO, and VLC benchmarks are slowed by $3-4 \times$. Hence, this application-specific tradeoff is worthy of exploring as a potential customization.

2) Multiplication Support: Whether multiplication is supported in hardware or software can greatly affect the area, performance, and power of a soft processor. There may be many variations of multiplication support, which trade area for cycle 


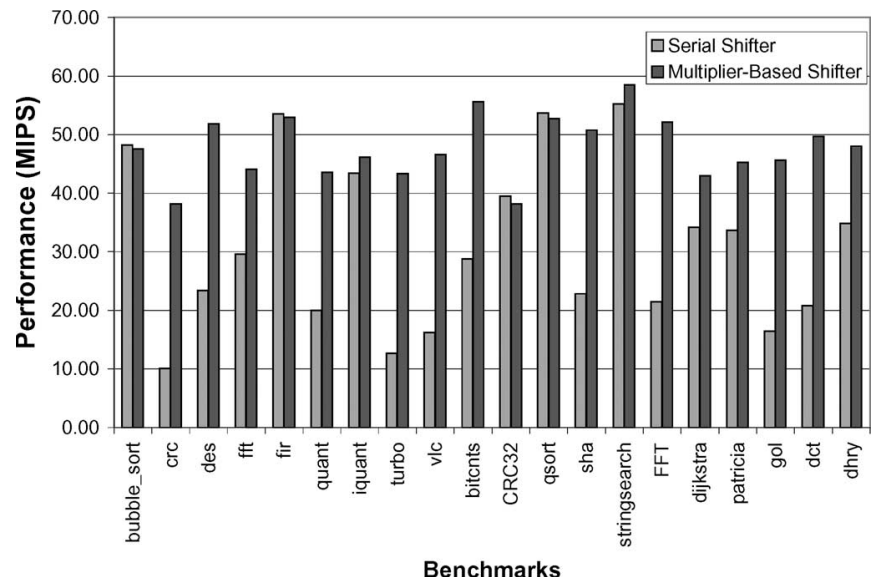

Fig. 3. Performance of a three-stage pipelined processor with two different shifter implementations.

time; we consider only full multiplication support using the dedicated multipliers in the FPGA. We make this simplification because we found that the hard multipliers on FPGAs are so area efficient that alternative implementations made of LUTs and flip-flops (whether booth, array, etc.) are consistently less efficient.

The area of the hardware multiplier is generally 230 equivalent LEs; however, only 160 of those are attributed to the actual multiplier. The remaining 70 LEs compose the glue logic required to hook the functional unit into the datapath including the MIPS HI/LO registers- the creators of the MIPS ISA separated the multiplier from the normal datapath by having it write its result to dedicated registers (HI and LO) instead of to the register file. This decision was later criticized [30], and we also find it to be a problem for FPGA designs: Since multiplication can be performed quickly in FPGAs (only one or sometimes two cycles longer than an adder), it does not require a special hardware to help overcome its cycle latency. Rather, the special registers and multiplexing prove to be wasted hardware, especially in the case of a shared multiplier/shifter, since the shift result must be written to the register file anyway (hence, the path from the multiply unit to the register file exists in spite of the attempts by the ISA to prevent it). We therefore agree with the approach taken in the Nios II ISA where separate multiply instructions compute the upper and lower words of the product.

Fig. 4 indicates that the performance of a processor that supports multiplication in hardware can vastly exceed one with only software multiplication support. Half of our 20 benchmarks do not contain multiplication, but for the other half, the results vary from suffering $8 \times$ more instructions executed as for IQUANT to an insignificant $1.8 \%$ increase for DIJKSTRA. Depending on the frequency of multiply instructions in the application, if any exist at all, a designer may look more favorably on the reduced area of a software implementation. We therefore deem multiplication support to be an important potential customization axis.

Our study of functional units has identified and quantified the hardware tradeoffs in implementing different shifter and multiplication support, which will both later be used to tune a processor to its application. In addition, we have pointed out the inappropriateness of MIPS to force separation of multi-

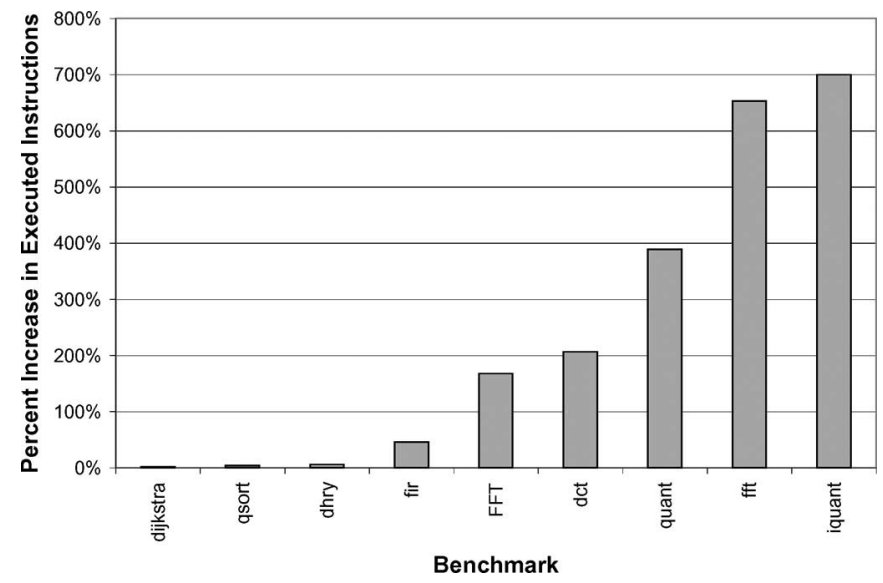

Fig. 4. Increase in total executed instructions when using a software multiplication subroutine instead of a single-instruction multiplication in hardware.

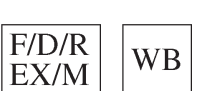

(a)

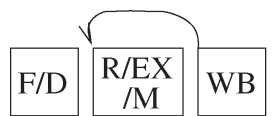

(b)

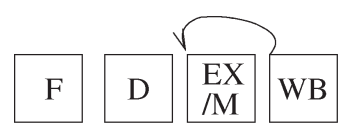

(c)

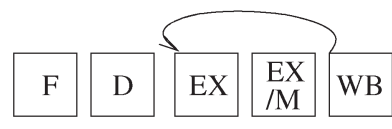

(d)

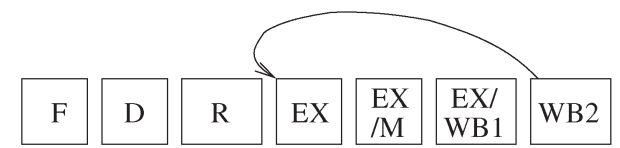

(e)

\begin{tabular}{|ll|}
\hline \multicolumn{1}{|c|}{ LEGEND } & \\
F - Fetch & Possible \\
D - Decode & Forwarding \\
R - Register & Path \\
EX - Execute & \\
M - Memory & \\
WB - Writeback & \\
\hline
\end{tabular}

Fig. 5. Processor pipeline organizations studied. Arrows indicate possible forwarding lines. (a) Two-stage. (b) Three-stage. (c) Four-stage. (d) Five-stage. (e) Seven-stage.

plies from the normal datapath through the use of the HI/LO registers: For FPGA-based designs where the multiplier is not dramatically slower than any other functional unit, this "special case" handling of multiplication is unnecessary.

\section{B. Pipelining}

We now use SPREE to study the impact of pipelining in softprocessor architectures by generating processors with pipeline depths between two and seven stages, the organizations of which are shown in Fig. 5. A one-stage pipeline (or purely unpipelined processor) is not considered since it provides no benefit over the two-stage pipeline. The writeback stage can be pipelined with the rest of the execution of that instruction for free, increasing the throughput of the system and increasing the size of the control logic by an insignificant amount. This free pipelining arises from the fact that both the instruction memory and register file are implemented in synchronous RAMs which require registered inputs. Note that we similarly do not consider 


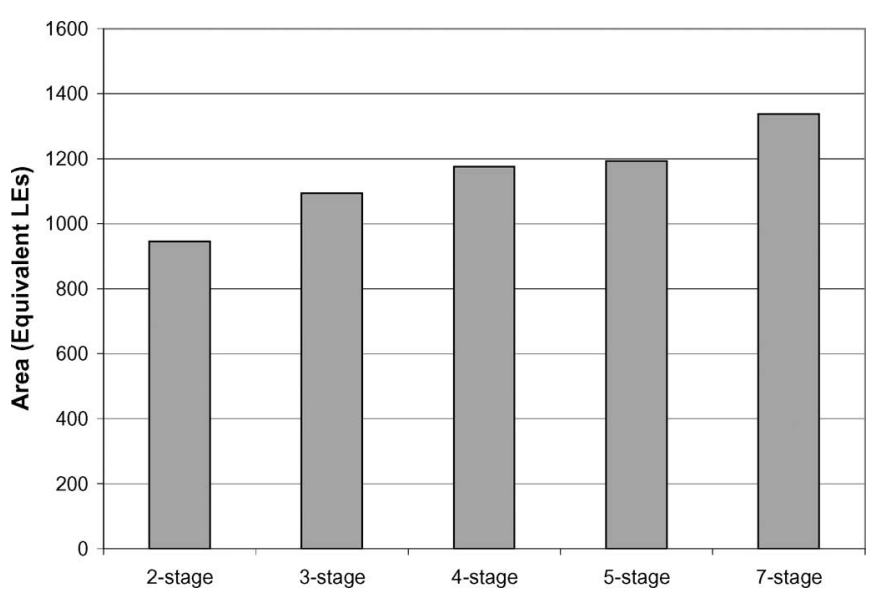

Fig. 6. Area across different pipeline depths.

a six-stage pipeline, since the five-stage pipeline has competing critical paths in the writeback stage and decode stage, which require both stages to be split to achieve a significant clockfrequency gain. For every pipeline, data hazards are prevented through interlocking, branches are statically predicted to be not taken, and misspeculated instructions are squashed.

1) Pipeline Depth: Fig. 6 shows (as expected) that the area increases with the number of pipeline stages due to the addition of pipeline registers and data hazard detection logic. However, we notice that the increase in area is mostly in combinational logic and not registers. Even the seven-stage pipeline has only a dozen LEs occupied with only a register, while 601 LEs are occupied without a register. Register-packing algorithms can typically combine these, but likely did not for performance reasons. As such, there is plenty space for the design to absorb flip-flops invisibly, since we expect register packing to place these in the 601 LEs occupied without a register. But, inserting these registers into the design breaks up logic into smaller pieces, which are less likely to be optimized into LUTs. This causes the combinational logic to be mapped into more LUTs, which increases area, along with the necessary data hazard detection and stalling/squashing logic which also contribute to the increased area.

Fig. 7 shows the performance impact of varying pipeline depth for four applications which are representative of several trends that we observed. The performance is measured in instruction throughput which accounts for both the frequency of the processor and its cycles-per-instruction behavior. The figure does not show the two-stage pipeline as it performs poorly compared to the rest. The synchronous RAMs in Stratix must be read from in a single stage of the pipeline for this design; hence, it suffers a stall cycle to accommodate the registered inputs of the RAM. The seven-stage pipeline also has a disadvantage: Branch delay slot instructions are much more difficult to support in such a deep pipeline, increasing the complexity of the control logic for this design. In contrast, the trends for the three-, four-, and five-stage pipelines vary widely by application. DES experiences up to $17 \%$ improved performance as the pipeline depth increases from three to five stages, while for STRINGSEARCH performance degrades by $18 \%$. SHA maintains consistent performance across the pipelines, which is a typical trend for many applications. For DHRY, the performance

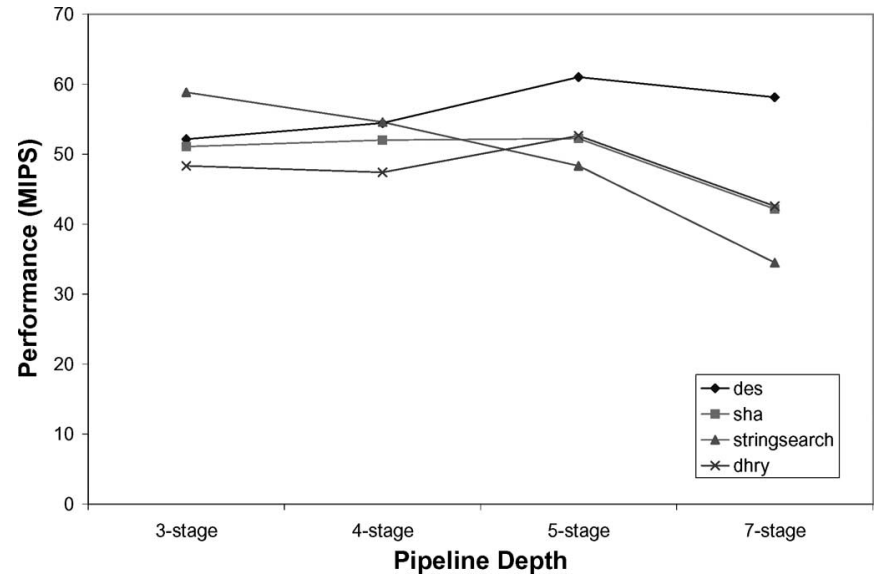

Fig. 7. Performance impact of varying pipeline depth for select benchmarks.

decreases by only $2 \%$ and then increases by $11 \%$. Pipeline depth is therefore another application-specific tradeoff, due to the fact that some applications suffer more than others from branch penalties and data hazards of varying distances.

For most individual benchmarks and when considering the average across all benchmarks, the pipelines perform the same for the three-, four-, and five-stage pipelines, while the sevenstage pipeline performs slightly worse for the reasons mentioned above. As such, we are inclined to conclude that the three-stage pipeline is most efficient since it performs equally well while using less area. We suspect that this is caused partly by the coarse-grained positioning of flip-flops and the large logic capacity of a LUT which is underutilized when there is little logic between registers. However, there is another factor to consider: There are many architectural features which SPREE does not currently support that could be added to favor the deeper pipelines, for example, better branch prediction and more aggressive forwarding. Nonetheless, this sentiment, that shorter pipelines are better, is echoed by Xilinx's Microblaze [2] which also has only three stages, and also Tensilica's Diamond 570T [7] processor which has only five stages (but is designed for an ASIC process).

2) Pipeline Organization: Tradeoffs exist not only in the number of pipeline stages but also in the placement of these stages. While deciding the stage boundaries for our three-stage pipeline was obvious and intuitive, deciding how to add a fourth pipeline stage was not. One can add a decode stage as shown in Fig. 5(c) or further divide the execution stage. We implemented both pipelines for all three shifters and observed that although the pipeline in Fig. 5(c) is larger by 5\%, its performance is $16 \%$ better. Hence, there is an area-performance tradeoff, proving that such tradeoffs exist not only in pipeline depth but also in pipeline organization.

3) Forwarding: We also examined the effect of implementing the forwarding paths shown in Fig. 5 either for both MIPS source operands, one of them, or none at all. Although not shown, we found that the variance in trends between applications for different forwarding paths is insignificant. We found that forwarding can provide area/performance tradeoffs in general, but none that differ significantly on a per-application basis. Typically, forwarding is a "win" for all applications sometimes providing $20 \%$ faster processors at the expense of 


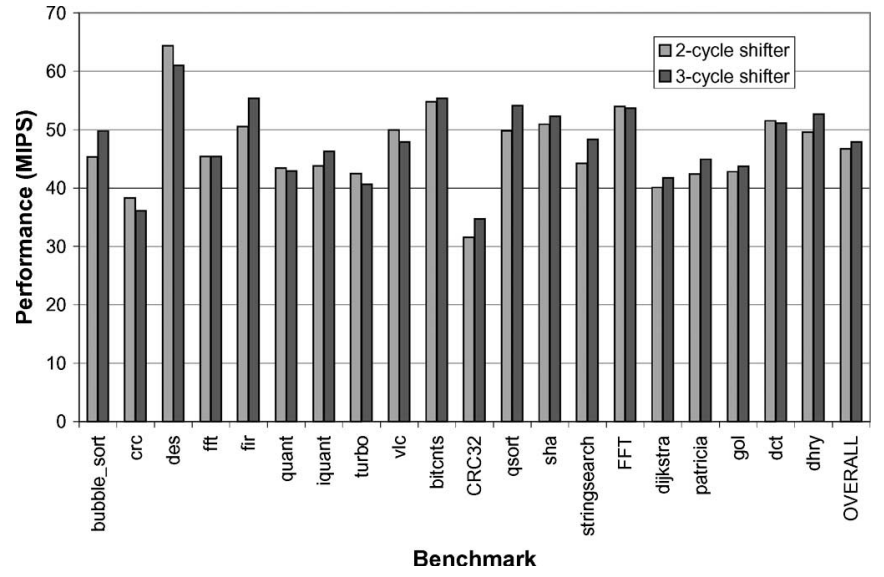

Fig. 8. Performance tradeoff in implementing unpipelined multicycle paths on a processor across the benchmark set.

approximately 100 LEs. While this result matches what is expected in ASIC-implemented processors, we project for deeper pipelines that more aggressive forwarding would result in more costly multiplexing, leading to unique FPGA-specific tradeoffs that differ from those of ASIC processors.

\section{Unpipelined Multicycle Paths}

Adding pipeline registers increases frequency but can also increase total CPI, as data hazards and branch penalties result in additional pipeline stalls. Alternatively, registers can be used in a more direct way for trading clock frequency and CPI. Registers can be inserted within a bottleneck pipeline stage that occasionally prevents that stage from completing in a single cycle, but that also allows the stage (and hence the entire pipeline) to run at a higher clock frequency. Moreover, with flip-flops readily available in every LE and embedded in the block RAMs and multipliers, this register insertion can come with only small area increases.

As a concrete example, we consider the five-stage pipeline with two-cycle multiplier-based barrel shifter. This processor has a critical path through the shifter which limits the clock speed to $82.0 \mathrm{MHz}$ while achieving 1.80 average CPI across the benchmark set. We can create another unpipelined multicycle path by making the multiplier-based shifter a three-cycle unpipelined execution unit which results in a clock frequency of $90.2 \mathrm{MHz}$ and 1.92 average CPI. The $10 \%$ clock-frequency improvement is countered by an average CPI increase of $6.7 \%$. Fig. 8 shows the instruction throughput in MIPS of both processors for each benchmark and indicates that benchmarks can favor either implementation. For example, BUBBLE_SORT achieves $10 \%$ increased performance when using the threecycle multiplier-based shifter while CRC achieves $6 \%$ increased performance with the two-cycle implementation. With respect to area, the two processors differ in area by only a single LE. Hence, we can use the unpipelined multicycle paths to make application-specific tradeoffs between clock frequency and CPI. Note that this technique is not limited to the execution stage, and it can be applied anywhere in the processor pipeline. In the set of explored processors, this technique was explored in large execution units (either the shifter or multiplier) whenever these units lay in the critical path.

\section{Impact of Customizing Soft Processors}

In this section, we use the SPREE system to measure the impact of customizing soft processors to meet the needs of individual applications. We demonstrate the impact of three techniques: 1) tuning the microarchitecture for a given application by selecting architectural features which favor that application but do no alter the ISA; 2) subsetting the ISA to eliminate hardware not used by the application (for example, if there is no multiplication, we can eliminate the multiplier functional unit); and 3 ) the combination of these two techniques.

\section{A. Application-Tuned Versus General Purpose}

We have demonstrated that many microarchitectural axes provide application-specific tradeoffs that can be tuned in soft processors to better meet the application requirements. In this section, we use SPREE to implement all combinations of these architectural axes - three shifter implementations, five pipeline depths, hardware/software multiplying, four forwarding configurations, two to three separately adjusted functional unit latencies, as well as miscellaneous pipeline organizations. We exhaustively search for the best processor for each application in our benchmark set. Specifically, we described each processor, generated it using SPREE, synthesized and placed and routed it using our CAD flow, and finally computed and compared the performance per area for each benchmark and processor pair. Performance per area is used as our metric since many of our architectural axes trade area and performance (for example, the benefit of using a serial shifter or software multiply is in reducing area at the expense of performance). We call the best processor the application-tuned processor, which ideally is the processor a designer (or intelligent software) would choose given the application and this set of processors. We also determine the processor that performed best on average over the complete benchmark set-this we refer to as the general-purpose processor. We then analyze the difference in efficiency between the general-purpose processor and the application-tuned processors and, hence, evaluate the potential for making application-specific tradeoffs in soft-processor microarchitecture.

Fig. 9 shows the measured efficiency in MIPS/LE in four bars: 1) The best on average (general-purpose) processor of those we generated using SPREE - this processor (the threestage pipeline with multiplier-based shifter) was found to provide the best geometric mean performance per area across the entire benchmark set; 2) the best per benchmark SPREE processor; 3 ) the best on average of the Nios II variations-experiment showed it was the Nios II/s; and 4) the best per benchmark of the Nios II variations from either Nios II/s, Nios II/e, or Nios II/f.

Focussing only on the SPREE processors in the first two bars, we noticed only six of the 20 benchmarks achieve their highest performance per area using the best overall processor; instead, the best processor for each benchmark varies and offers significantly better efficiency. By choosing an 


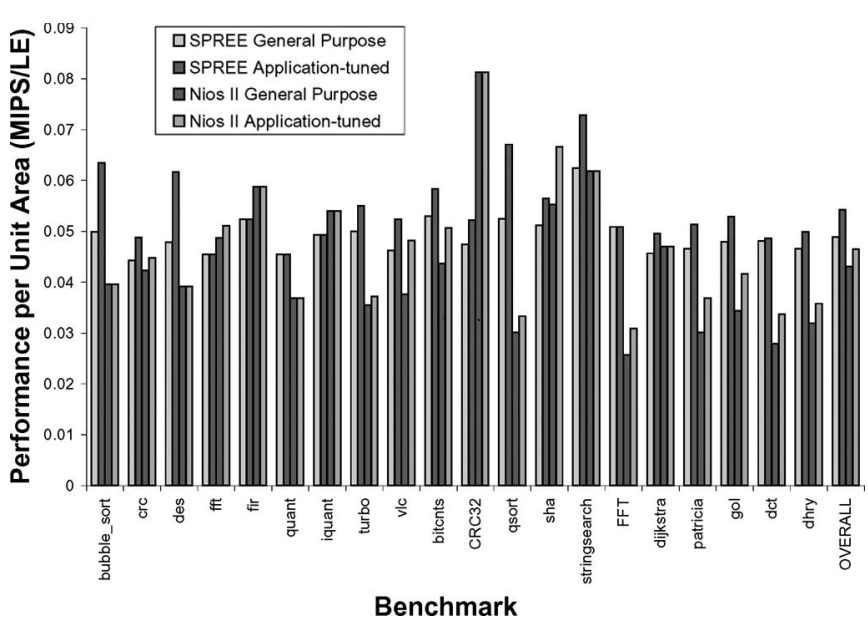

Fig. 9. Performance per area for each benchmark for SPREE (i) best on average (general-purpose) processor and (ii) best per benchmark (applicationtuned) processor as well as for Nios II (iii) general purpose and (iv) application tuned.

application-tuned processor, the average performance per area is improved by $14.1 \%$ over the best overall processor across the entire benchmark set; furthermore, STRINGSEARCH, QSORT, CRC32, and BUBBLE_SORT improve the performance per area by approximately $30 \%$. The results indicate that these ISAindependent modifications made in the processor core can be substantial and are certainly worth pursuing in a system-onprogrammable-chip platform where general-purpose efficiency is not a key design consideration and the reconfiguration of the processor is free. In future work, we expect this benefit to increase significantly when supporting more advanced architectural axes such as datapath widths, branch predictors, aggressive forwarding, caches, and very long instruction words (VLIWs).

The best per-benchmark processors had many predictable trends. Benchmarks without significant shifting benefited from a smaller serial shifter, benchmarks with little or no multiplying utilized software multiplication instead of a multiplier functional unit. Full forwarding on both operands was always present except for three benchmarks which chose no forwarding. In terms of pipeline stages, all benchmarks used three-stage pipelines except for three (a different triplet than those with no forwarding) which used five-stage pipelines likely due to reduced stress on the pipeline allowing those benchmarks to enjoy the higher frequencies. In the future, we hope to select the appropriate architecture from analysis of the application.

We now compare our results with the Nios II processor variations shown in the latter two bars. For most of the benchmarks, the application-tuned SPREE processors yield significantly better performance per area than any of either the generalpurpose SPREE processor or the commercial Nios II variants. On average across all benchmarks, the application-tuned processors generated by SPREE are the most efficient, yielding $16 \%$ better results than Nios II because of the more fine-grained customization space afforded by SPREE - the Nios II actually spans a larger design space than the SPREE with the Nios II/e being smaller and the Nios II/f being faster than any current SPREE processor. This leads us to suggest that many fine-grain microarchitectural customizations can yield better efficiencies

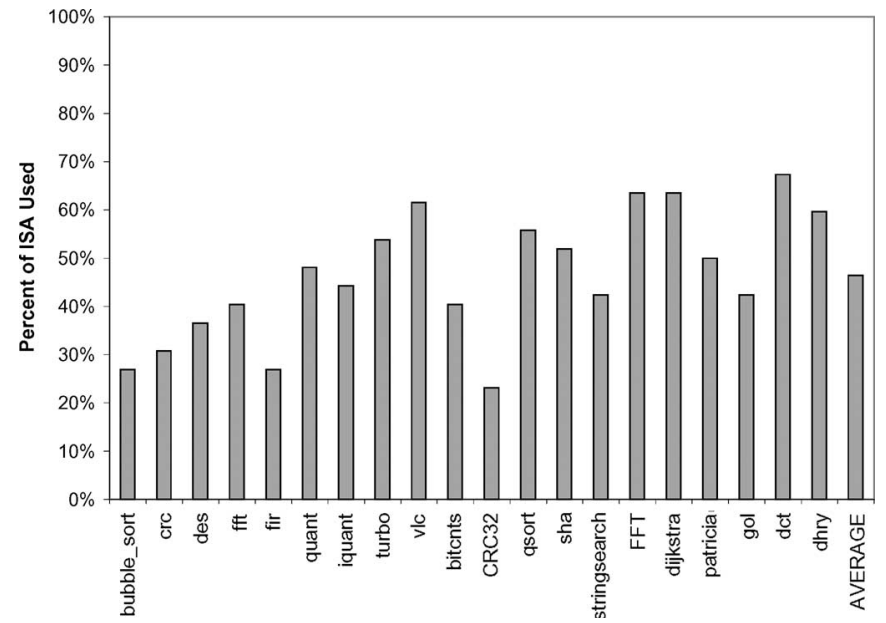

Fig. 10. Instruction set utilization across benchmark set.

than a few separate hand-optimized cores targeting specific design space points.

\section{B. ISA Subsetting}

So far, we have investigated the microarchitectural customizations that favor an individual application but still fully support the original ISA. In this section, we propose to capitalize on situations where: 1) only one application will run on the soft processor and 2) there exists a reconfigurable environment allowing the hardware to be rapidly reconfigured to support different applications or different phases of an application. We customize the soft processor by having it support only the fraction of the ISA, which is actually used by the application. SPREE performs this ISA subsetting by parsing the application binary to decide the subsetted ISA, removing unused connections and components from the input datapath, and then generating simpler control. Fig. 10 shows the fraction of the 50 MIPS-I instructions supported by SPREE that are used by each benchmark, which is rarely more than $50 \%$. BUBBLE_SORT, finite-impulse response (FIR), and CRC32 use only about one quarter of the ISA. With such sparse use of the ISA, we are motivated to investigate the effect of eliminating the architectural support for unused instructions.

To evaluate the impact of ISA subsetting, for each of the 20 benchmarks, we subsetted three processor architectures: 1) a two-stage pipeline with LUT-based barrel shifting; 2) the three-stage pipeline with multiplier-based barrel shifting; and 3) a five-stage pipeline with LUT-based barrel shifting. Note that the processors with LUT-based shifting are somewhat contrived since having the separate large LUT shifter will emphasize the effects of subsetting; however, such a situation may arise in low-end devices which do not contain dedicated multipliers. All three processors utilize hardware multiplication support. Since the cycle-by-cycle execution of each benchmark is unaffected by this experiment, we use a clock frequency to measure the performance gain.

The relative area of each subsetted processor with respect to its nonsubsetted version is shown in Fig. 11. In general, on our best processor, the three-stage with multiplier-based shifting, we can expect $12.5 \%$ area reductions. The three benchmarks 


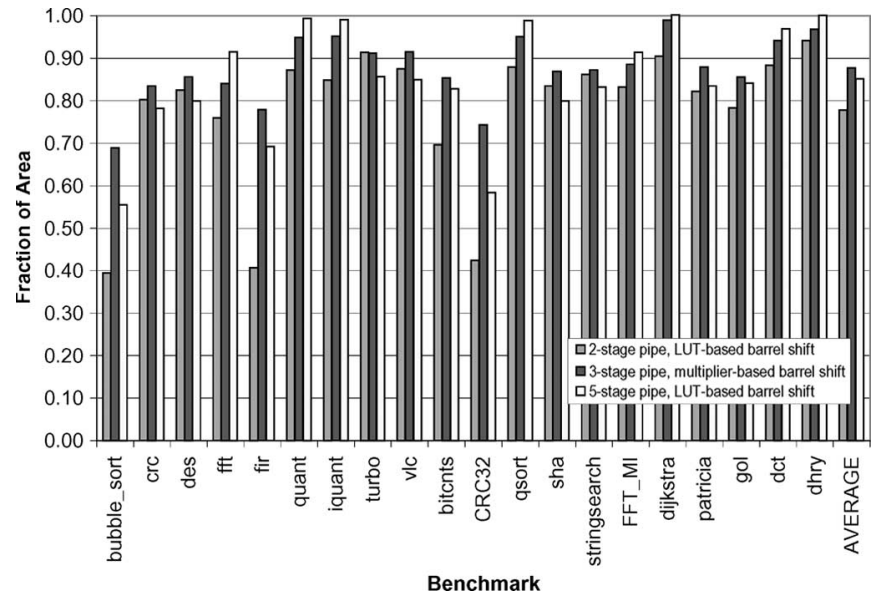

Fig. 11. Impact on area of ISA subsetting on three architectures.

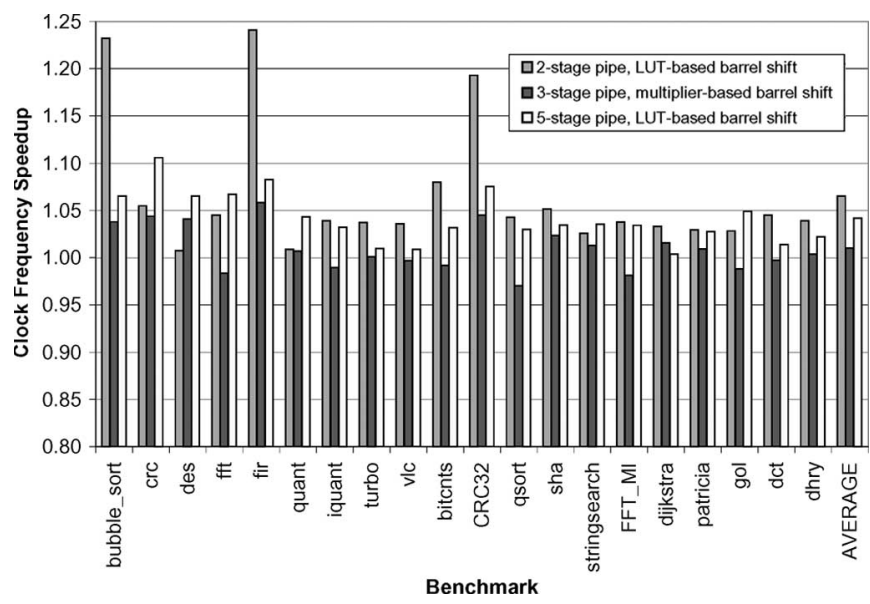

Fig. 12. Impact on clock speed of ISA subsetting on three architectures.

which use only $25 \%$ of the ISA (BUBBLE_SORT, FIR, and CRC32) obtain the most significant area savings. For the contrived two-stage architecture, these three benchmarks obtain $60 \%$ area savings, while most other benchmarks save $10 \%-$ $25 \%$ area. Closer inspection of these three benchmarks reveals that they are the only benchmarks which do not contain shift operations-shifters are large functional units in FPGAs, and their removal leads to a large area savings. However, the MIPS ISA obstructs such a removal because there is no explicit nop instruction; instead, nops are encoded as a shift left by zero. Therefore, to remove the shifter, one must include special hardware to handle these nop instructions, or else reencode the nop. In this paper, nops are reencoded as add zero (similar to Nios II) to allow for complete removal of the shifter, since all benchmarks use adds. The savings are more pronounced in the two- and five-stage pipelines where the shifter is LUT based and hence larger.

Fig. 12 shows the clock-frequency improvement for the subsetted architectures. In general, we see modest speedups of $7 \%$ and $4 \%$ on average for the two- and five-stage pipelines, respectively. The three-stage pipeline is not improved at all, as its critical path is in the data hazard detection logic and, hence, cannot be removed. More positively, the modest clockfrequency speedups indicate that our pipelines have wellbalanced logic delays: When logic is removed from a given

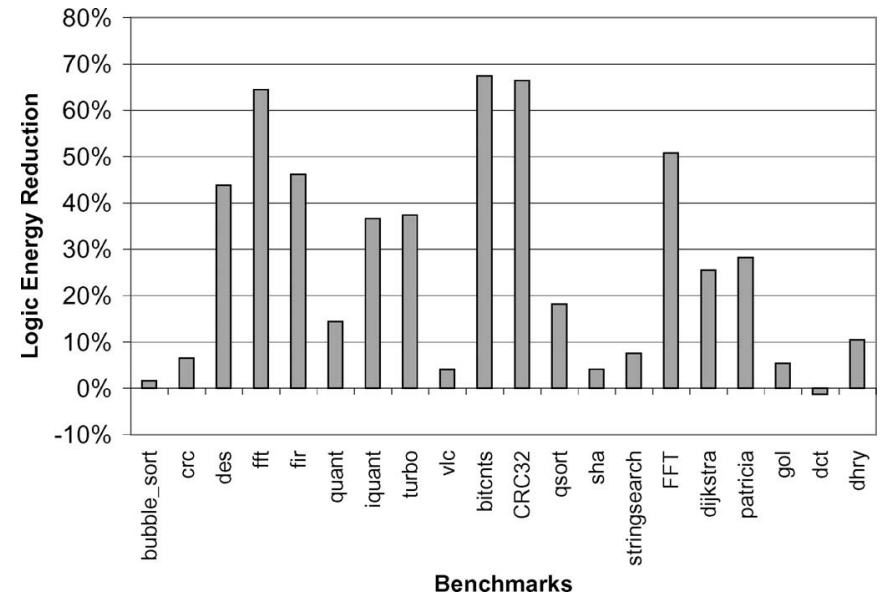

Fig. 13. Energy reduction of subsetting on three-stage pipeline.

path, there is often another path to maintain the previous critical path length; hence, the odds of a given subsetting reducing all paths are relatively small. However, there is a notable performance improvement in the two-stage pipeline for the three benchmarks without shifts, BUBBLE_SORT, FIR, and CRC 32 . This is because the LUT-based shifter lays in the critical path of the pipeline, and caused poor balancing of logic delay. Removing the shifter allows for a roughly $20 \%$ improvement in the clock frequency for these benchmarks.

Fig. 13 shows the reduction in energy resulting from ISA subsetting. Note that the energy dissipated by the memory is ignored since it accounts for $80 \%-90 \%$ of the energy, and it can never be removed by subsetting since all applications fetch and write to memory. The figure demonstrates that the removal of high-toggle rate components and simplified control results in significant energy savings in the processor pipeline. The subsetted processors of some benchmarks such as FFT, BITCNTS, and CRC 32 provide greater than $65 \%$ energy savings. On average across all the subsetted processors, approximately $27 \%$ of the nonmemory energy can be saved. A miniscule increase in energy was seen for the DCT benchmark, which we attribute to noise in the CAD system; total system energy decreased very slightly but the fraction attributed to logic increased unexpectedly.

\section{Combining Customization Techniques}

We have presented two methods for creating applicationspecific soft processors: 1) architectural tuning, which alters soft-processor architecture to favor a specific benchmark and 2) ISA subsetting, which removes architectural support that is not utilized by the benchmark. In this section, we compare the effectiveness of the two techniques in terms of performance per area both individually and combined. We define the best general-purpose processor as the single processor which achieves the greatest performance per area on average across all benchmarks, and the best application-tuned processors as the set of processors which achieve the best performance per area for each benchmark. For each processor and benchmark, we then perform the ISA subsetting and measure the performance per area of the four combinations: general purpose, application 


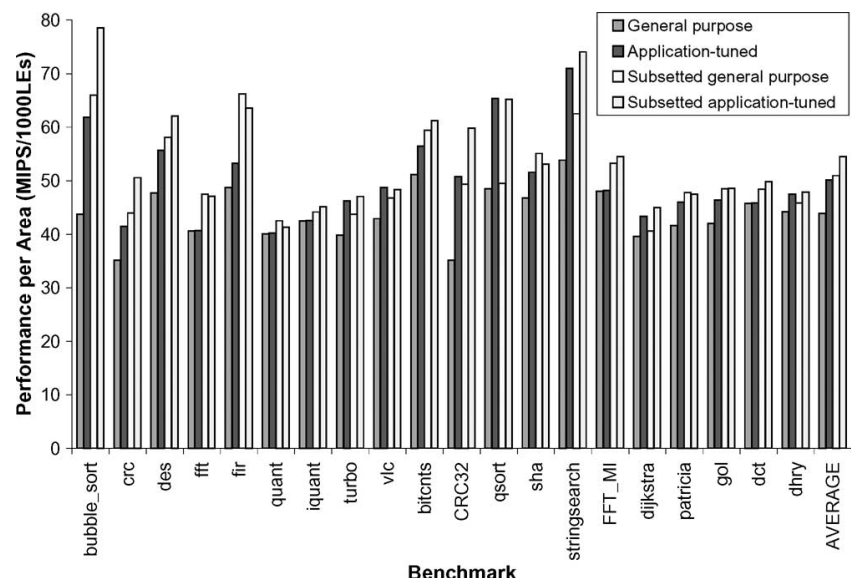

Fig. 14. Performance per area of tuning, subsetting, and their combination.

tuned, subsetted general purpose, and subsetted application tuned.

Fig. 14 shows the performance per area for all four combinations. As shown previously, the application-tuned processor is consistently better than the general-purpose processor. ISA subsetting is more effective on the general-purpose processor than on the application-tuned processors: The performance per area is improved by $16.2 \%$ on average for the general-purpose processor while by only $8.6 \%$ for the application-tuned processor. This is intuitive since the hardware which was eliminated during subsetting was likely reduced in size during the tuning of the application-tuned processor. For example, FIR uses no shifting; therefore, a small serial shifter is chosen during tuning and later removed in subsetting, resulting in a less dramatic area reduction. There is a large variation when deciding between these two methods: some benchmarks such as FIR achieve up to $25 \%$ increased performance per area by using the applicationtuned processor over the subsetted general-purpose processor, while others such as QSORT achieve a $25 \%$ increase by using the subsetted general-purpose processor over the applicationtuned processor (i.e., they are opposite). These two methods are very competitive as summarized in Fig. 14 by the AVERAGE bars, which show the subsetted general-purpose processor with slightly higher performance per area than the application tuned (by only $2.2 \%$ ).

The subsetted application-tuned processor combines all customizations (both the microarchitectural tuning and the ISA subsetting) and therefore often achieves the highest performance per area. In some cases, a single technique actually does better, but closer inspection reveals that these are typically a result of the inherent noise in CAD algorithms. For example, for FIR subsetting, the general-purpose processor achieves a frequency improvement of $5.5 \%$ while subsetting the tuned architecture actually resulted in a frequency decrease of $3.2 \%$ which when combined created a less-efficient processor in spite of any area savings. The combination of the two techniques is mostly complementary. On average, subsetted applicationtuned processors achieve more than $10 \%$ better performance per area across the benchmark set than either microarchitectural tuning or ISA subsetting alone. However, for each benchmark, either technique on its own can come to within $4 \%$ of the combined approach. Overall, the combined approach can im- prove the performance per area by $24.5 \%$ on average across all benchmarks.

\section{CONCLUSION}

The reconfigurability of soft processors can be exploited to meet design constraints by making application-specific tradeoffs in their microarchitecture, a method that requires a complete understanding of soft-processor microarchitectures and how they perform/map on/to FPGA devices. In this paper, we use the soft-processor RTL implementations generated by the SPREE infrastructure to study the soft processors on both of these fronts: 1) we explore the influence of this new FPGA hardware platform on the tradeoffs within soft-processor microarchitecture and 2) we explore the impact of customizing soft processors to their applications including through the use of our ISA subsetting technique which removes all hardware not required by the application.

Using our SPREE soft processor generator, we explored tuning the microarchitecture by selecting the best processor from a large number of processor variants for a specific application. We determined that the best of these applicationspecific processors offers considerable advantages over the best on average general-purpose processor, an improvement in performance per area of $14.1 \%$ on average across all benchmarks. This significant efficiency improvement will likely increase as we consider additional architectural axes in future work. Also, we saw that the wide range of processors provided by SPREE were more efficiently mapped to applications than the three Nios II variants which span a larger design space.

Finally, we used the SPREE infrastructure to perform ISA subsetting, where for each application, the hardware support for unused features of the ISA is removed. With this technique, we obtain large reductions in the area and power of the resulting processors, reductions of approximately $25 \%$ for each metric on average and up to $60 \%$ for some applications. Combining our techniques for microarchitectural tuning with the ISA subsetting results in an even more dramatic benefit where the performance per area is improved by $24.5 \%$ on average across all applications.

In the future, we will explore a more broad set of customizations including branch prediction, caches, datapath width, VLIW datapath parallelism, and other more advanced architectural features. We also plan to investigate more aggressive customization of these processors, including changing the ISA to encourage customization. Finally, we are interested in exploring the benefits of tuning the compiler based on exact knowledge of the target architecture.

\section{REFERENCES}

[1] Nios II, Altera. [Online]. Available: http://www.altera.com/products/ip/ processors/nios2

[2] MicroBlaze, Xilinx. [Online]. Available: http://www.xilinx.com/ microblaze

[3] J. Turley, "Survey: Who uses custom chips," Embedded Systems Programming, vol. 18, no. 8, Aug. 2005.

[4] I. Kuon and J. Rose, "Measuring the gap between FPGAs and ASICs," in Proc. FPGA, 2006, pp. 21-30.

[5] P. Yiannacouras, J. Rose, and J. G. Steffan, "The microarchitecture of FPGA based soft processors," in Proc. Int. Conf. CASES, 2005, pp. 202-212. 
[6] P. Yiannacouras, J. G. Steffan, and J. Rose, "Application-specific customization of soft processor microarchitecture," in Proc. Int. Symp. FPGA, 2006, pp. 201-210.

[7] D. Goodwin and D. Petkov, "Automatic generation of application specific processors," in Proc. Int. Conf. Compilers, Architectures and Synthesis Embedded Syst., 2003, pp. 137-147.

[8] J. M. Arnold, "S5: The architecture and development flow of a software configurable processor," in Proc. Int. Conf. FPL, Dec. 2005, pp. 121-128.

[9] J. R. Hauser and J. Wawrzynek, "Garp: A MIPS processor with a reconfigurable coprocessor," in Proc. IEEE Symp. FPGAs Custom Comput. Mach., K. L. Pocek and J. Arnold, Eds., 1997, pp. 12-21. [Online]. Available: http://www.citeseer.ist.psu.edu/hauser97garp.html

[10] P. Biswas, S. Banerjee, N. Dutt, P. Ienne, and L. Pozzi, "Performance and energy benefits of instruction set extensions in an FPGA soft core," in Proc. 19th Int. Conf. VLSID, 5th Int. Conf. Embedded Syst. Des., 2006, pp. 651-656.

[11] R. Lysecky and F. Vahid, "A study of the speedups and competitiveness of FPGA soft processor cores using dynamic hardware/software partitioning," in Proc. DATE, 2005, pp. 18-23.

[12] R. Dimond, O. Mencer, and W. Luk, "CUSTARD-A customisable threaded FPGA soft processor and tools," in Proc. Int. Conf. FPL, Aug. 2005, pp. 1-6.

[13] M. Itoh, S. Higaki, J. Sato, A. Shiomi, Y. Takeuchi, A. Kitajima, and M. Imai, "PEAS-III: An ASIP design environment," in Proc. Int. Conf. Comput. Des., Sep. 2000, pp. 430-436.

[14] P. Mishra, A. Kejariwal, and N. Dutt, "Synthesis-driven exploration of pipelined embedded processors," in Proc. VLSI Des., 2004, pp. 921-926.

[15] G. Braun, A. Nohl, W. Sheng, J. Ceng, M. Hohenauer, H. Scharwachter, R. Leupers, and H. Meyr, "A novel approach for flexible and consistent ADL-driven ASIP design," in Proc. 41st Annu. DAC, 2004, pp. 717-722.

[16] K. Keutzer and M. Gries, Building ASIPs: The Mescal Methodology. New York: Springer-Verlag, 2005. [Online]. Available: http://www. gigascale.org/pubs/603.html

[17] G. Gibeling, A. Schultz, and K. Asanovic, "RAMP architecture description language," in Proc. Workshop Architecture Res. Using FPGA Platforms, 2006, pp. 4-8. Proc. 12th Int. Symp. on High-Performance Computer Architecture.

[18] J. Gray, Designing a Simple FPGA-Optimized Risc CPU and System-ona-Chip, 2000. [Online]. Available: http://www.citeseer.ist.psu.edu/article/ gray00designing.html

[19] P. Yiannacouras, "The microarchitecture of FPGA-based soft processors," M.S. thesis, Univ. Toronto, Toronto, ON, 2005. [Online]. Available: http://www.eecg.toronto.edu/ jayar/pubs/theses/Yiannacouras/ PeterYiannacouras.pdf

[20] — SPREE. [Online]. Available: http://www.eecg.utoronto.ca/ $\sim$ yiannac/SPREE/

[21] MINT Simulation Software. (1996, Jan. 13). Univ. Rochester, Rochester, NY. [Online]. Available: http://www.cs.rochester.edu/u/veenstra/

[22] D. M. Lewis et al., "The Stratix routing and logic architecture," in Proc. Int. Symp. Field-Programmable Gate Arrays, 2003, pp. 12-20.

[23] R. Cliff, Altera Corporation, Private Communication, 2005.

[24] M. Guthaus et al., "MiBench: A free, commercially representative embedded benchmark suite," in Proc. IEEE 4th Annu. Workshop Workload Characterisation, Dec. 2001, pp. 3-14.

[25] A. Lodi, M. Toma, and F. Campi, "A pipelined configurable gate array for embedded processors," in Proc. ACM/SIGDA 11th Int. Symp. FPGA, 2003, pp. 21-30.

[26] Dhrystone 2.1. (1988, May 25). Freescale. [Online]. Available: http:// www.freescale.com

[27] RATES-A Reconfigurable Architecture TEsting Suite. (2003, Mar. 27). Univ. Toronto Toronto, ON, Canada. [Online]. Available: http://www. eecg.utoronto.ca/ lesley/benchmarks/rates/
[28] Stratix Device Handbook. (2005, Jul.). Altera, San Jose, CA. [Online] Available: http://www.altera.com/literature/lit-stx.jsp

[29] P. Metzgen, "Optimizing a high-performance 32-bit processor for programmable logic," in Proc. Int. Symp. Syst.-on-Chip, 2004, p. 13

[30] J. Mashey, "Internet nuggets," Comput. Archit. News, vol. 32, no. 4, pp. 1-14, Sep. 2004

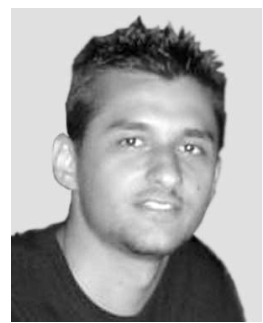

Peter Yiannacouras (S'06) received the B.A.Sc. degree from the Engineering Science Program at University of Toronto, Toronto, ON, Canada, and the M.A.Sc. degree from the Electrical and Computer Engineering Department at the same university, where he is currently working toward the Ph.D. degree.

He has also worked with Intel Microarchitecture Research Labs. His research interests include processor architecture, embedded processing, fieldprogrammable gate array (FPGA) logic architecture, and automatic customization

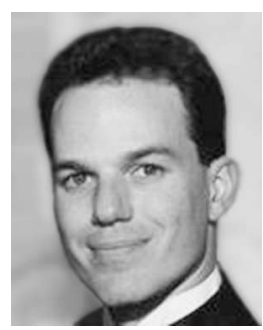

J. Gregory Steffan (S'93-M'03) received the B.A.Sc. and M.A.Sc. degrees in computer engineering from University of Toronto, Toronto, ON, Canada, in 1995 and 1997, respectively, and the $\mathrm{Ph} . \mathrm{D}$. degree in computer science from Carnegie Mellon University, Pittsburgh, PA, in 2003.

He is currently an Assistant Professor of computer engineering with University of Toronto. He has also worked with the architecture groups of millions of instructions per second (MIPS) and Compaq. His research interests include computer architecture and compilers, distributed and parallel systems, and reconfigurable computing.

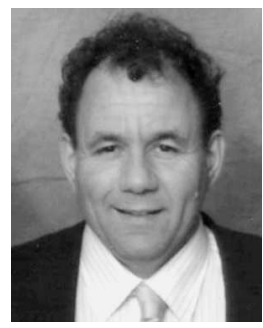

Jonathan Rose (S'86-M'86-SM'06) received the $\mathrm{Ph} . \mathrm{D}$. degree in electrical engineering from the University of Toronto, Toronto, ON, Canada, in 1986.

He was a Postdoctoral Scholar and then a Research Associate in the Computer Systems Laboratory at Stanford University, from 1986 to 1989. In 1989, he joined the faculty of the University of Toronto. He spent the 1995-1996 year as a Senior Research Scientist at Xilinx, in San Jose, CA, working on the Virtex field-programmable gate-array (FPGA) architecture. $\mathrm{He}$ is the co-founder of the ACM FPGA Symposium and remains part of that Symposium on its steering committee. In October 1998, he co-founded Right Track CAD Corporation, which delivered architecture for FPGAs and packing, placement, and routing software for FPGAs to FPGA device vendors. He was President and CEO of Right Track until May 1, 2000. Right Track was purchased by Altera and became part of the Altera Toronto Technology Centre. His group at Altera Toronto shared responsibility for the development of the architecture for the Altera Stratix, Stratix II, Stratix GX, and Cyclone FPGAs. His group was also responsible for placement, routing, delay-annotation software, and benchmarking for these devices. From May 1, 2003 to April 30, 2004, he held the part-time position of Senior Research Scientist at Altera Toronto. He has worked for Bell-Northern Research and a number of FPGA companies on a consulting basis. He is currently a Professor and Chair of the Edward S. Rogers, Sr., Department of Electrical and Computer Engineering, University of Toronto. 